\title{
Study on Artistic Characteristics of Shandong Clapper Ballad
}

\author{
Xuemei Chen \\ School of Music and Dance \\ Qujing Normal University \\ Qujing, China 655011
}

\begin{abstract}
S handong clapper ballad, the treasure of Chinese folk performing art, has obvious regional characteristics and it is an intangible cultural heritage at national level and one of the leading fork performing arts of Chinese fork art big family. Making its future from Legend of Wu Song, through unremitting efforts made by generations of predecessor masters and artists, Shandong clapper ballad is inherited generation after generation, endless stream of pilgrims, influencing over the country, even overseas. To date, it has been listed in the first batch of nonmaterial cultural heritage list. Over the years, Shandong clapper ballad has won praise from the public, not only as the local folk custom of Shandong but also the inheritance of culture. This paper mainly researches on the artistic characteristics of Shandong Clapper ballad and introduces its historical origin, inheritance and development and representative schools in detail. In addition, it details the Gao school, Yang school and Liu school with their artistic characteristics analyzed and explains the characteristics of Shandong clapper ballad by selecting representative periods to allow people to know it and lay certain theoretical foundation for the artistic inheritance and development of Shandong clapper ballad.
\end{abstract}

Keywords-Shandong clapper ballad; Chinese folk performing art; school; artistic characteristics

\section{INTRODUCTION}

On May 20, 2006, Shandong clapper ballad was normally included in the first batch of nonmaterial cultural heritage of China. At the earliest, it was evolved from the legend of $\mathrm{Wu}$ Song, who was also known as Wu Laoer due to his ranking the second at home. Also because $\mathrm{Wu}$ Song the protagonist had a big and tall figure, many people called it sing the big one. Later, a great number of folk art forms appeared with it in different periods, e.g. bamboo clapper ballad and funny ballad. In June 1949, Gao Yuanjun, a Shanghai artist, normally denominated the record of $L u \mathrm{Da}$ Eradicates the Bully as Shandong Clapper Ballad in the performance link of the Greater China.

As of the middle 20th century, thanks to Gao Yuanjun together with the folk performance teams cultivating lots of professional performers and putting them into Shandong clapper ballad, it has made large differences in spreading scope. Till the middle and later periods of 20th century, more and more powerful performers have appeared in China, including Liu Sichang, Sun Zhenye and Gao Jingzuo, etc.
However, in the meanwhile, some famous actresses who had unique styles and wide influence appeared as well. From then, Shandong clapper ballad became more and more popular.

\section{DEVELOPMENT HIST ORY OF SHANDONG CLAPPER BALLAD}

\section{A. Historical Origin of Shandong Clapper Ballad}

At the earliest, Shandong clapper ballad was evolved from the folk traditional arts of Han Chinese, experiencing a cultural history of over 100 years. Originally, it was popular in Shandong area, northern China and Northeastern China region and gradually spread over the country till peaceful liberation. In general, the clapper ballad performers hold the bamboo clappers clapping in hand to perform, hence, it is also known as bamboo book.

According to investigation, Shandong clapper ballad is originated from Linqing and Jining regions that artists say Shandong clapper ballad comes from river which not means general rivers but the ancient canal connecting the north and south. Linqing and Jining, as the big wharfs of the canal, are the gathering places of travelers and businessmen always on the move, which commonly known as Fertile Soil. That very year, talking-singing arts thrived, which provided a favorable location condition for the breeding and formation of Shandong clapper ballad. The last years of the Northern Song, Linqing and Jining were right the places where the heroes in Liangshan bustled and the tale of the hero $\mathrm{Wu}$ Song were widely circulated at that time. Hence, Shandong clapper ballad emerged at the right moment with the spread of the Legend of Wu Song.

\section{B. Inheritance and Development of Shandong Clapper Ballad}

Prior to the peaceful liberation of China, the Chinese Shandong clapper ballad was only spread in rural areas at the earliest; however, with the constant development of the society, it entered into urban areas gradually. From the perspective of region, it can be divided into in-province and out-province two forms. Within the province, Shandong was a city formed through slow expansion from Lucia areas and Jinan was the capital of Shandong province. In Yantai, Qingdao and other places of Shandong Province, various 
artic traces of artists existed, among which, Qingdao region had the most. Shandong clapper ballad gradually became famous because of Gao Yuanjun and Yang Lide. In addition to these two talents, the existence of a great number of similar artists was common, whose strength should not be overlooked. Outside the province, it developed southeastwards along the Yangzi River and no Shandong capper ballad appeared until the end of 1930's in Wuhan, Xuzhou, Bengbu, Nanjing, Shanghai and other areas. Qi Yongli and Gao Yuanjun all started from Shandong capper ballad at that time. Of which, Gao Yuanjun was best at the performance time and he was praised "popular in Jiangnan". At this point, Shandong capper ballad stepped onto the historical stage of cultural art forms of our country.

\section{REPRESENTATIVE SCHOOLS OF SHANDONG CAPPER BALLAD}

Based on telling and singing, Shandong capper is characterized by strong rhythm of language and basic sentence pattern of seven-word sentence of "two-two-three" that generally it end in three words to ensure lively rhythm and beats two same copper plate (Yuanyang plate) with left hand as musical instrument accompaniment. At the earliest, Shandong capper ballad has two schools of Gao Yuanjun and Yang Lide; and Liu Sichang risen later established his own school. The following is the introduction to Gao school, Yang school and Liu school as well as their art achievements.

\section{A. Gao School and Its Characteristics}

The appearance of Gao Yuanjun in Shandong clapper ballad allows a wider propagation of Shandong drum and makes a great difference and Gao possesses a lot of students with high achievements. He is characterized by vividness, truth, goodness and interesting. Yang Lide once said that Gao Yuanjun is full of energy in his performance style and draws multiple advantages from opera. He acts a lot, even too much. In the work of Discussion on the characteristics of Gao Yuanjun Shandong Clapper Ballad, it mentioned that in the performance of Shandong clapper ballad, Gao Yuanjun is realistic in art, and by talking and singing, he molded various artistic images of figure. His representative works include $L u$ Da Eradicates the Bully, Li Kui Seizes Fish, Zhao Kuangyin Roars Majiadian as well as the A Cartful of Sorghum Rice, The Scout and the Behead Luo Ping by Strategy, etc. among which, the most significant representative performance is the full-length Shandong clapper ballad Legend of Wu Song.

\section{B. Yang School and Its Characteristics}

Yang Lide was born in Shandong Province. His clapper ballad is full of the special charm of Shandong and possesses special style. He pays much attention to the skills of enunciation and strictly requires that the style of clapper ballad should be full of humor and lightness. And there should be no extra moves. Therefore, his clapper ballad finally becomes unique in his style which is called the "Yang School". "Qiao" is developed based on the plot of story. The long sentences can consist of ten sentences or eight sentences which will make the rhythm more clear. On the aspect of singing, it can not only well describe the scene but also should express the role which will bring the feeling of enjoying of arts. Yang Lide is also good at performing the Legend of Wu Song and once created the new shows of Shandong clapper ballad such as Hao Huaiyou and Quqiangji, etc and wrote the book of How to Perform Shandong Clapper Ballad with Zhang Jun.

\section{Liu School and Its Characteristic}

The so-called Liu School is led by Liu Sichang. The essence of Liu school is mainly manifested in realism. Its performance behaviors can show the current social life and can shape the artistic images full of vivid details. What's more, the Liu school is no being performed in many places. In addition, the school also possesses the performance skills such as singing, ventriloquism, movie, drama, acrobatics and oven the ballet and those skills are slowly introduced to the Shandong clapper ballad which enriches the performance forms and manifests the brand-new level of Shandong clapper ballad. These are the contributions that Liu school made to Shandong clapper ballad. His master works are Red Courtyard, Fishing for Bronze Bull, Drung Ridge, The Section Chief, Miro the Black Child, Tiger in Zhayi, Listening to the Radio, Learning Foreign Language, Transferring and Premier Zhou Riding a Same Car with Us, etc and he also created more than one hundred of works of Chinese folk art forms in other forms which mainly are monochord of Sister and Brother, catchword repetition clapper ballad of Song Enzhen, story-telling in Beijing dialect with drum accompaniment of Three Sisters, a variety of Dagu popular in Hebei and Henan provinces of Cui's Kiln, allegro of Song of Lei Feng, Henan Zhuizi of Sister Ran, comic dialogue of Blind Copying and make-up comic dialogue of Riding Electric Cars (cooperate with other persons), etc.

\section{ARTIST IC CHARACTERIST ICS OF SHANDONG CLAPPER BALLAD}

The Shandong clapper ballads are all the form of standing singing and stress the application of "hands, eyes, body and steps" and the "humor" and "link points" in the performance. The singing words are basically the sevenword sentences and the performers will sing the dialogs with spoken parts in the middle. While the forms of programs have "single paragraph", "Chang-shu" and "Shu-mao", etc. Because the Shandong clapper ballads are performed by one or several performers by using simple dramatis props, it can obtain good artistic effects in a sudden. But also because it is not limited to the places, no matter it is the edge of a field, construction sites, station, wharf and streets and lanes, it can be performed at any time which can reflect the real life fast and serve the economic construction. Therefore, it can be enduring for several hundred of years and owns a broad mass base. Many classic stories have been broadly spread among the mass and enjoyed great love. The author will introduce several artistic characteristics of Shandong clapper ballad below. 


\section{A. Link Points" That Can Draw Attraction}

The Shandong clapper ballad is classified as art in the folk art world. In fact, some points need to be generated from the plots. As for the virtual links, usually the details come first following with the plots in the measure of self-deception. Those are what are appreciated in the Shandong clapper ballad. Those are closely related with what is thought of great brilliance by the audiences. For example, in the Shandong clapper ballads, the Legend of Wu Song is formed by depending on many different stories. Usually, each story is set with one link point and will take one day to be performed. That is the Shandong clapper ballad. The story plots are also very close. Besides, its language possesses the measure of being concise and humorous. We should say that the story starts with link point. For many works, the audiences would image when will the fight start, how will they fight and what is the result of fighting after watching. It objectives are to satisfy the audiences of different age grades and meet the expectation of audiences. Everyone who has listened to the Shandong clapper ballad would know if you listen carefully, you will not be thinking of leaving, because the links in Shandong clapper ballad are closed connected.

\section{B. Emphasis on the Vibration of Rhythm of Language}

The so-called clapper ballad of Gao school not only stresses the language skills of being delayed and fast with pause and inflexion, but also depends on the measure of hitting allegros for the narration on the aspect of language. Its objective is the vibrations on the rhythm of language which consists of the types of singing of being fast, slow, strong, weak, delayed, intensive, pause and inflexion. It also uses beautiful singing to promote the atmosphere, reflect the vibration of emotion, describe figures and give the audiences the sense of beauty.

\section{Creative Intensive Hitting and Slow Singing}

Attention should be paid to the performance methods of the traditional ballads is to stress the nature. However, it is relatively monotonous on the aspect of rhythm. It will be hard for the performance method to show highlights when the conditions of vibrations on events or of complicated moods occur. For example, there is a part of compliment of the tiger in Wu Song Fighting with Tiger: there are one black line and one yellow line on its body. There is also one bloody mouth on it. If the two eyes of it widely open, they would be like the mug. There is the character of Wang on its forehead. Wu Song fixed his eyes on it and it turned out to be a real tiger and Wu Song was wet with cold sweat under the shocking.

\section{Passionate and Unconstrained Plots}

The Shandong clapper ballad formulates a series of stories about $\mathrm{Wu}$ Song, the hero and the plots of stories are also very moving. And we can also see such moving plots in our real life very often.

For example, the chapters of Temple of God of Mount Tai disclose the behaviors of bullies like five brothers of $\mathrm{Li}$ family who were finally eradicated by $\mathrm{Wu}$ Song.

\section{E. One Performer for One Play}

On the aspect of performance, the Shandong clapper ballad doesn't need scenery and doesn't need any props and actors as the accompaniment. One performer stands on the entire stage, with two cooper (steel) allegros in left hand and another pair of bamboo clappers in right hand. And the sounds generated from hitting: the sound of cooper clappers are Dang-li-ge-dang; the bamboo clappers are Kuang-kunagkuang. On the stage, the performer must be standing and will sing while walking. There should also be enough space for giving play. Now the bamboo clappers have been abandoned and the right hand is freed. Therefore, it will be more convenient for the performers. There are many roles in the book.

All the things will be accomplished by one person. No matter for the sound or action, the one person will accomplish all by him or herself and should be vivid during performance, which is a tough task. It is exactly because it is hard that the performers must practice with efforts. They must perform well to ensure the audiences to obtain the satisfactory experiences.

\section{CULTURAL CHARACTERISTICS OF SHANDONG CLAPPER BALLAD}

\section{A. Full-Bodied Local Flavor}

Shandong clapper ballad differs very much on the performance measures. In the past performance, we can say that the Shandong clapper is not polite enough. Because on the aspect of clothing, it adopted the half-naked dressing measure for the performance. So metimes, the singing words were not so civilized. So metimes, there are even swearwords So many ungenteel behaviors were not only closely related to the life of the mass at that time, but also would make the audiences feel closer. And on the performance actions, the scale will be gradually changed. Later, at the time of national great liberation, the Shandong clapper ballad also gradually ascends on the national stage and those uncivilized words are also deleted. But the characteristics on language will still be reserved. And the Shandong clapper ballad will still e mit the folk flavor.

\section{B. Art Staying Close with the Trend of the Times}

Many people are saying that the current Shandong clapper ballad has been replaced. However, I think that this opinion is relatively opinionated. On the world, changes are occurring on many things. The Shandong clapper ballad is the artistic form of long standing and it possesses profound foundation. For example, the development speed of Shandong has been continuously changing. For example, the stories such as A Cartful of Sorghum Rice, Capturing Prisoners and Quqiangji and other stories all possess great potential and the possibility for future development. Therefore, on the art of Shandong clapper ballad, it is still very promising. 


\section{CONCLUSION}

As the contemporary young people, we should realize that the Shandong clapper ballad is not just a kind of historical and cultural heritage but is more like a kind of valuable resource. And we should actively advocate and support the current situation and the inheritance of Shandong clapper ballad. As the intangible cultural heritage, we should focus on the issues such as how to protect the characters of our culture under the current economic globalization. It is not only the direct manifestation of national personality, but also is the important carrier of the national sentiments and national cohesion. The reasons why the Shandong clapper ballad is that famous are that it possesses special artistic character and possesses special artistic charm. Therefore, only if we can fully give play to its artistic style and continue to inheriting and promoting its styles, can we certainly reserve the Shandong clapper ballad in the arts of intangible cultural heritages permanently so as to keep the roots of our culture, envis age the historic glory and revive the power of culture.

\section{REFERENCES}

[1] Wang Wenzhang. Introduction to Intangible Cultural Heritage[M] Beijing: Education Science Press, October, 2008

[2] Liu Yan. Discussion on the Changes on Arts of Shandong Clapper Ballad in Contemporary Society [J]. Song of the Yellow River, 2009, (19): 66-67.

[3] Wang Guizeng. How the Shandong Clapper Ballad is Defined[J]. Zhongshanfengyu Press, 2008, (04): 64

[4] Li Hailiu. Investigation on the Historic Origin of Shandong Clapper Ballad of Gao School[J]. Oriental Collection, 2012, (03): 108-109.

[5] Li Xiangjun. Shandong Clapper Ballad: Rich Themes- Forever Shining[J]. 2004, (8): 70-71.

[6] Wang Li. Artistic Characters of Shandong Clapper Ballad[J]. Drama Collection, 2010, (4): 126-128.

[7] Zhang Jun. Creation and Performance of Shandong Clapper Ballad[J]. Shandong People's Publishing House, 1981, (9): 32-34.

[8] Gao Yuanjun. Discussion on the Arts of Shandong Clapper Ballad[J]. People's Literat ure Publishing House, 1982, (5): 61-62. 